\title{
A ZigBee Wireless Sensor Network for Monitoring an Aquaculture Recirculating System
}

\author{
Francisco J. Espinosa-Faller*, Guillermo E. Rendón-Rodríguez \\ Universidad Marista de Mérida, Periférico Norte Tablaje 13941, Mérida, Yucatán, 97300, México. \\ *fjespinosa@marista.edu.mx.
}

\begin{abstract}
A ZigBee wireless sensor network was developed for monitoring an experimental aquaculture recirculating system. Temperature, dissolved oxygen, water and air pressure as well as electric current sensors were included in the setup. The high fish densities required in these systems to become economically viable present a case where sensor networks can be applied to preserve a healthy livestock and to reduce the risk of failures that end up in the loss of production. Modules for reading and transmitting sensor values through a ZigBee wireless network were developed and tested. The modules were installed in an aquaculture recirculating system to transmit sensor values to the network coordinator. A monitoring program was created in order to display and store sensor values and to compare them with reference limits. An alert is emitted in case reference limits have been reached. E-mail and an SMS message alert can also be sent to the cellular phone of the system administrator, so immediate action can be taken. A web interface allows Internet access to the sensor values. The present work demonstrates the applicability of ZigBee wireless sensor network technology to aquaculture recirculating systems.
\end{abstract}

Keywords: Wireless sensor networks, ZigBee, aquaculture monitoring systems.

\section{RESUMEN}

Se desarrolló una red de sensores inalámbrica con el protocolo ZigBee para monitorear un sistema experimental acuícola con recirculación de agua. La red incluye sensores de temperatura, oxígeno disuelto, presión de agua y aire, así como de corriente eléctrica. La alta densidad de organismos requerida para que estos sistemas sean económicamente viables nos presentan un caso donde las redes de sensores pueden ser aplicadas para preservar un stock de peces saludable reduciendo las probabilidades de fallas que conlleven pérdidas en la producción. Se desarrollaron y probaron módulos para la toma y transmisión de datos a través de una red ZigBee y se implementaron en una granja acuícola experimental. Se desarrolló un programa de monitoreo para desplegar los valores de los sensores y emitir alertas cuando se rebasen los límites de referencia especificados. Una alerta por medio de un mensaje SMS y un correo electrónico pueden ser emitidas. Una interfase WEB permite el acceso a los valores de los sensores. El presente trabajo demuestra la aplicabilidad de las redes inalámbricas ZigBee al monitoreo de sistemas acuícolas.

\section{Introduction}

Aquaculture research has focused on using less freshwater in intensive sustainable production systems. Aquaculture recirculating systems are characterized by high fish densities with minimal effluent discharge $[1,2]$. In general, they consist of circular tanks in which settable solids are flushed through a drain. Solids are then captured in a filtering unit and the water is passed through a biological filter for ammonia removal and an ultraviolet unit for disinfection. Finally the water returns to the tanks to complete a cycle. Dissolved oxygen level in the water is sustained through the injection of air coming from a blower.
High fish densities introduce new challenges for the optimal control of the system parameters. Response times in case of a system failure have to be fast enough to preserve livestock. The availability of dissolved oxygen, which is in general the most critical parameter in terms of response time makes the monitoring procedure a task that optimally should be performed continuously. A real time monitoring system for some critical parameters can even lead to improvements in productivity and quality and could even add value to the final product, by certifying the production process history [3]. 
Monitoring systems, wired and wireless, exist today in industry. They are based on standards that can be implemented in aquaculture recirculating systems (For example, the Hart protocol, www.hartcomm.org, the YSI5200 system, www.ysi.com, or the OneWireless Network from Honeywell Process Solutions hpsweb.honeywell.com). However, new technologies, specifically wireless, are being developed [4-6]. Those wireless technologies can be adapted and used successfully in aquaculture systems and fish farms [7-13].

Wireless sensor network research has been an active topic in the last years [14-19]. As a result, standards have emerged in order to commercialize the technology (For example, the ZigBee Alliance and the 6LowPAN) $[14,15]$. Standardization of the physical (radio) level and network layers is leading to a wide spread availability of low-cost system-on-chip devices and modules. Among wireless network standards, ZigBee specializes in low-cost, low-power and short range communications based on the IEEE 802.15.4 standard for the physical (PHY) and medium access control (MAC) layers [15]. In addition to the PHY and MAC layers the ZigBee standard adds application support and network layers, together with security and power management routines.

The multi-hop capability of a ZigBee network provides a method to extend the range and enhance reliability in the communications. The ZigBee standard is a low bit-rate communications protocol operating at $250 \mathrm{~kb} / \mathrm{s}$ (in the $2.4 \mathrm{Ghz}$ band). The main applications of the standard are designed for home and building automation and utility meter communication. ZigBee works in the unlicensed industrial, scientific and medical bands of $2.4 \mathrm{GHZ}, 900 \mathrm{MHz}$ and $868 \mathrm{Mhz}$.

ZigBee technology can provide a low-cost and easy deployment monitoring technology to be used in high fish density aquaculture. An additional advantage is that the wireless nature of the network makes it simple to change sensor positions depending on monitoring requirements and adjustments as the biomass of the systems increases together with fish grow [19]. Network transmission speed and response time if fast enough for this application since notification of abnormal events to the managers can be done in a few seconds after the failure event. In this work, we developed low-cost modules and wireless sensor network based on the ZigBee standard in order to monitor an aquaculture recirculating system.

\section{Materials and Methods}

\subsection{System overview}

The data acquisition system consists of wireless modules that read sensor information at scheduled times. The data is transmitted to the router or the coordinator which extracts the message body from the data packet and transmits the sensor values to the central PC computer (CPC) through a serial interface. The CPC monitoring program displays the incoming data, sends it to the database for storage and compares the values with reference limits for each sensor. In case a reference limit has been reached, the program sends automatic warnings through e-mail and/or cell phone SMS messages to the system administrator. The status of the system can also be monitored through a web page which displays the latest values for each sensor. Database mining can be performed through a web page linked to the monitoring web page. Figure 1 shows a representation of the system with the modules that collect and send the data to the router or coordinator and the coordinator that receives data and sends it through a serial RS-232 interface to the main computer for database storage. A web page server is implemented to monitor the system remotely. Also, e-mail and cell phone alerts are implemented. Details of the design and implementation of these components follow.

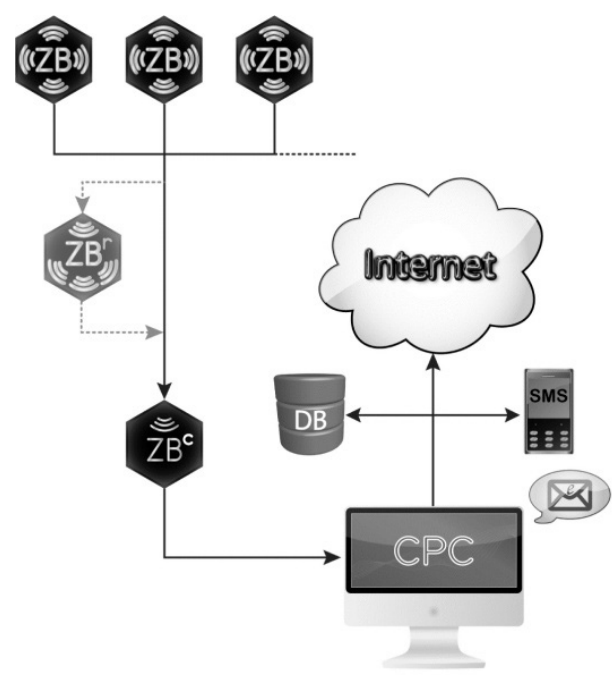

Figure 1. System configuration showing the data flow from the ZigBee modules (ZB) to the coordinator (ZBc), the central PC computer (CPC), the database (DB), the e-mail and warning messages (SMS) and the Internet. Optionally, a router can be included $(\mathrm{ZBr})$ to extend the network range or to include more devices. 


\subsection{Modules}

The modules are the elements that read sensor values and send the data to the coordinator through the wireless network. They were designed and assembled as part of this work and consist of a printed circuit board (PCB) with electronic components in a water tight box. Figure 2 shows the module assembly. We note that the PCB and the battery holder are mounted in such a way that no components are above or below the chip antenna and the connectors fit in the plastic box in a position close to the external connector to form a compact assembly.

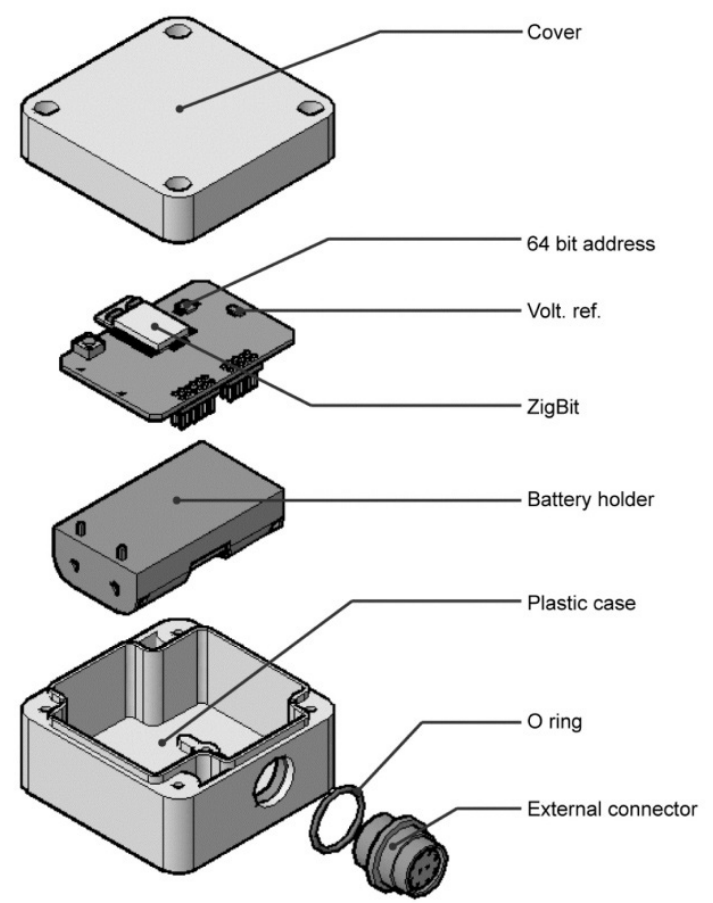

Figure 2. Components and assembly of the ZigBee module.

The electronic board consists of a ZigBit $2.4 \mathrm{GHz}$ integrated circuit assembly with a dual chip antenna from Atmel Corp. (ATZB-24-A2), a LT1790BCS6-1.25 voltage reference from Linear Technology Corp., and a DS2411P+ unique 64-bit IEEE address chip from Maxim Integrated Products, Inc. The electric diagram is shown in Fig. 3. The low-power nature of the ZigBit, consuming only microamperes in sleep mode and $\sim 18 \mathrm{~mA}$ in transmit mode, makes them a good solution for low-power wireless applications. The ZigBit module contains an ATmega1281V microcontroller, an AT86RF230 radio transceiver and a dual chip antenna. The voltage reference chip provides the reference for the analog to digital controller of the ZigBit's microcontroller and the 64bit IEEE address chip provides a unique address for each of our modules using a 1-wire interface to the microcontroller [20]. The module can be powered by two AA batteries or regulated 3VDC. A 10-terminal connector provides access for programming the module using a JTAG interface and a second connector in the sensor board provides access to the analog and digital $1 / O$ lines of the module.

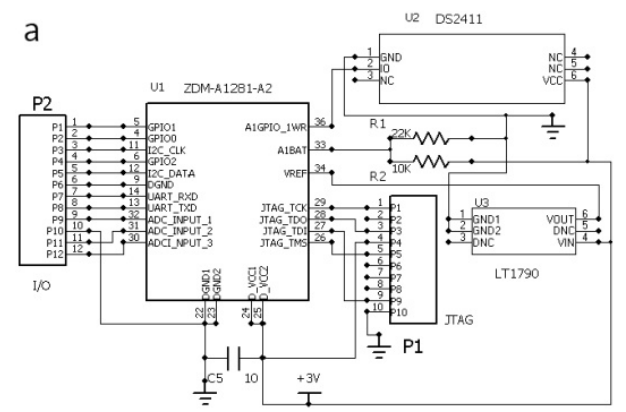

b

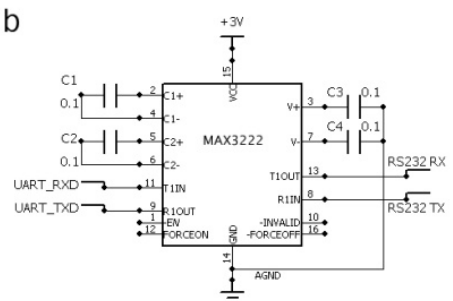

Figure 3. Electric diagram of the ZigBee wireless module. (a) End device; (b) RS-232 leve I converter for the coordinator

The circuit board is enclosed in a NEMA-4 type box (2.5" x 2.5" Hammond manufacturing Inc.), which has a PX0412/10S Bulgin connector to be able to work in the humid environment of the aquaculture research facility. Figure 4 shows the uncovered assembled module. The sensors are either directly connected to the module (the sensor board) or are electrically conditioned in a separate board and enclosure. The components are 
assembled in a printed circuit board designed to use only one copper layer. This design provides a low-cost module capable of forming a complex ZigBee network.

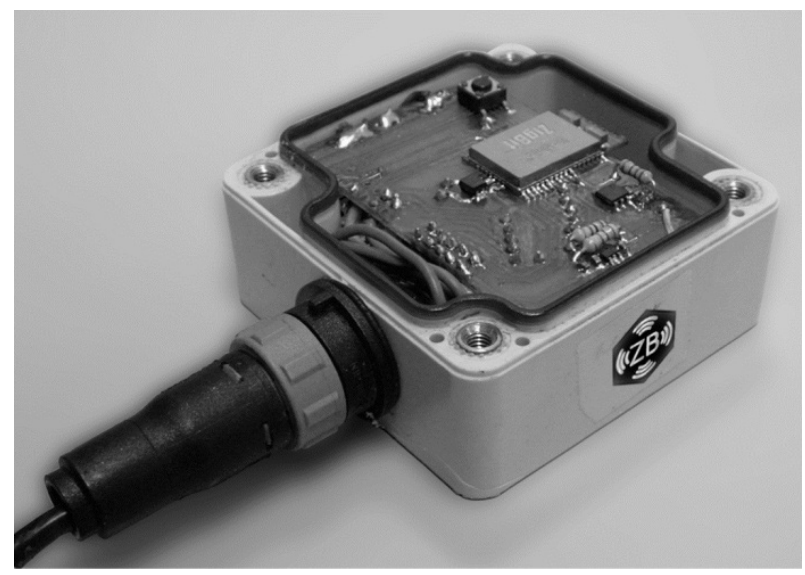

Figure 4. Uncovered module with the circuit board and external connector to the sensors.

The software to run the modules was developed in the $C$ programming language using the AVR-GCC compiler and the BitCloud ZigBee stack from ATMEL [21]. The source code can be requested to the authors as well as the board layout. The modules work in the following way: At startup, the module starts a procedure to find a parent node, either a router or a coordinator. When connected to its parent, the module starts its routine of sending the 10-bit voltage values of the sensors connected to its inputs every five seconds. After sending its data, the module goes to sleep and enters a low-power mode to save power. During sleep mode, the power consumption is only 0.43 $\mathrm{mW}$, while when transmitting the power increases to $5.4 \mathrm{~mW}$. After a specified wakeup time, the cycle starts again reading the voltages at its inputs and sending the new data. In case the network is lost for any reason, the module automatically starts looking for a parent node (a router or a coordinator) until it finds one.

\subsection{Coordinator}

The coordinator is identical to the modules in its hardware with the exception that it has a serial RS232 level converter (MAX3222 from Maxim, shown in Fig. 3b) in order to be able to connect our modules to the CPC using an RS-232 serial port.
Due to the nature of its task, the coordinator cannot sleep and enter into a low-power mode, so it has to be mains powered all the time. The software in the coordinator is programmed in the $\mathrm{C}$ programming language under the BitCloud stack [21]. When the coordinator is turned on, it starts a ZigBee network and listens for signals from modules that want to join. After the coordinator acknowledges a module with the proper credentials, using the ZigBee protocol, it registers the module as a member of the network and starts listening for incoming data. When a data packet is received, the coordinator extracts the message from the packet and forwards it to the CPC through a serial RS-232 interface.

The coordinator is programmed to handle up to 7 modules. If more modules are required, a router can be included as one of the child of the coordinator in order to handle 7 modules making a network of 13 modules, one router and one coordinator. The router automatically transfers the received messages to the coordinator and can be used not only to increase the number of nodes in the network but also to extend the range of the network by linking devices that in other way cannot reach the coordinator. The process of including routers can continue to include hundreds of devices if needed. The programming of the routers is also done in the $\mathrm{C}$ programming language under the ATMEL BitCloud ZigBee Stack and the hardware is identical to the modules. The router, as the coordinator, needs also to be awake all the time hence it needs to be mains powered. The low power of the modules limits the range of communication to $27 \mathrm{~m}$, as tested in our facilities; however as mentioned before, this can be overcome by including routers or by using amplified radio transceivers.

\subsection{Monitoring program}

The CPC runs the monitoring program (MP) that receives the data from the coordinator. First, the MP saves the data received in the database, displays the data in the monitor and in an instrument panel. Figure 5 shows a screenshot of the instrument panel of the MP. The program compares the received data with reference limits for each measured parameter and if a limit is reached, it can send an e-mail and an SMS cell phone message to the system administrator. All 
parameters in the monitoring program are configurable. Sensor calibration is also performed in the MP. The program was developed using the C\# programming language to run on a Windows PC.

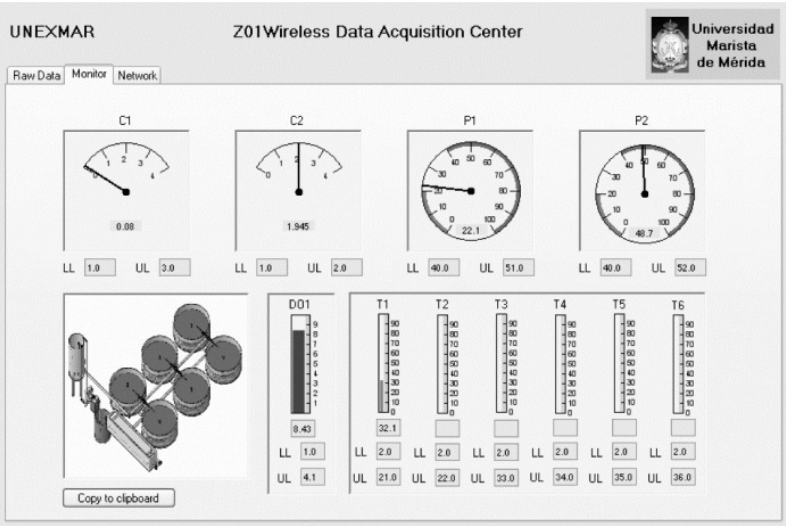

Figure 5. Instrument panel of the monitoring program.

\subsection{Database and Web}

The database for storing sensor values consists of one table per sensor where the table stores the index, date, time and sensor value. The MP can be configured to store data every selected time interval in minutes. The database is also the link between the MP program and the web page. The web page displays an instrument panel with the latest values stored in the database and automatically updates to display the new values. A database mining interface is also provided to retrieve the data from the selected sensor. The database used in this work was MySQL and the web page and data mining program was programmed using HTML, PHP and AJAX.

\subsection{Aquaculture research facility, system assembly and test}

The aquaculture research facility consists of a 144 $\mathrm{m}^{3}$ recirculating water system with 6 fish circular tanks. Water flows from the center of the tanks to a sedimenter to remove solids. The water is then pumped to a biological filter; it passes through a UV disinfection unit and returns to the tanks to complete the cycle. Dissolved oxygen in the tanks is enhanced through air diffusers connected to a $1 \mathrm{Hp}$ blower. A photograph of the aquaculture facility is shown in Figure 6 where module positions are indicated by arrows.
A D06421 dissolved oxygen sensor from Sensorex and a TMP37 temperature sensor from Analog Devices were installed in one of the six tanks of the aquaculture research facility. The output of the temperature sensor was connected directly to the module while the dissolved oxygen sensor was connected to a $64 \mathrm{ohm}$ resistor that converts the 4-20 mA output of the sensor to a voltage for the module. A water pressure sensor (Honeywell SPT4V0030PG5W02) was connected at the base of the biological filter and an air pressure sensor (Honeywell ASDX005G24R) was connected to the air pipe. Both signals were passed through resistors to decrease its maximum output of 5 Volts to the 1.2 Volts used as voltage reference for the analog to digital converter of the ZigBit. Electrical current in the recirculating pump was monitored through a Hall-effect current sensor (Honeywell CSLA2CD) whose sinusoidal voltage output signal was rectified and conditioned using a passive low pass filter to enter the modules [22].

The modules were installed about $1 \mathrm{~m}$ above the fish tanks with steel poles and in the structure that supports the biological filter (See inset of Figure 6). The coordinator was mounted on a specific post with clear sight of all the modules. The system was tested connecting the sensors to the modules and the coordinator to the CPC.

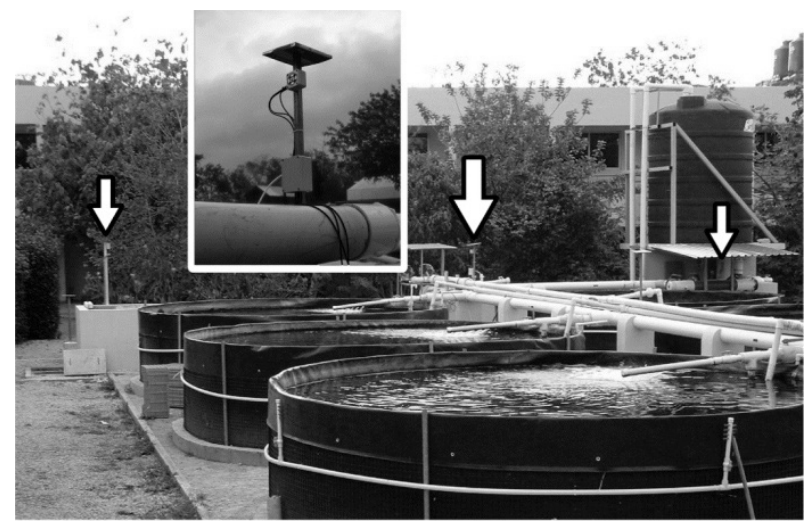

Figure 6. Aquaculture research facility. Arrows show modules positions. Inset: Module mounted on a post

\section{Results and discussion}

Data from the sensors is analyzed by the monitoring program and when a limit is reached in the incoming data the program sends a warning 
through an e-mail and an SMS message as described before. Also, the data can be visualized through the instrument panel in the CPC or from the web interface. The system administrator can analyze all the received information and get the warnings if something goes wrong even when out of the office or while in travel. A monitoring system like this can help in the event of a failure to take rapid actions to mitigate the damage that can be generated to the fish stock and the production facility.

The system has been working for more than six months, with or without rain, and in a hot and humid weather that has reached a temperature of $40{ }^{\circ} \mathrm{C}$ at midday. It should be noted that natural or artificial barriers such as trees or walls decrease the link quality between the modules and the network coordinator. For such cases, it might be necessary to use amplified radio interfaces (e.g., ZigBit modules with amplified RF signal).

Incoming data from the monitoring system is shown in Fig. 7. It is interesting to observe the dissolved oxygen graph (Fig. 7a). At night, the dissolved oxygen remains basically constant sustained by the water aeration, but during the day, photosynthesis from algae increments gradually the amount of dissolved oxygen to get a maximum at midday. Some of the features of the dissolved oxygen graph can be related to the feeding of the fish like the decrease at about $2 \mathrm{pm}$. In Fig. 7b, the pressure from the air blower for the water aeration shows a drop between 05:00 pm and 07:00 pm. The reason was a scheduled maintenance of the air pipes that reduced aeration. This reduction is then reflected in the dissolved oxygen (Fig 7a) giving a minimum at 07:00 pm, showing that the monitoring system actually reflects the real-time behavior of the aquaculture facility and that maintenance and other activities that perturb the facility can be monitored. Figure 7c shows the temperature of the water. Exposure to sunlight slowly increases temperature of the water while after sunset heat slowly diffuses out of the geo-membrane plastic fish tanks decreasing the temperature.

The information of dissolved oxygen levels could be used in the future to obtain energy savings in the system by using the 1HP water aeration blower only when it is needed, depending on the real time signal coming from the dissolved oxygen sensor.
Furthermore, the air pressure (Fig. 7b) could also be used to generate energy savings: since the dissolved oxygen in the water depends on water aeration, the rate of water aeration could be controlled through the air pressure using a proportional control to the blower. By implementing a control system, it is possible to regulate the dissolved oxygen in the water from the signal of the sensors and regulate the air pressure accordingly.
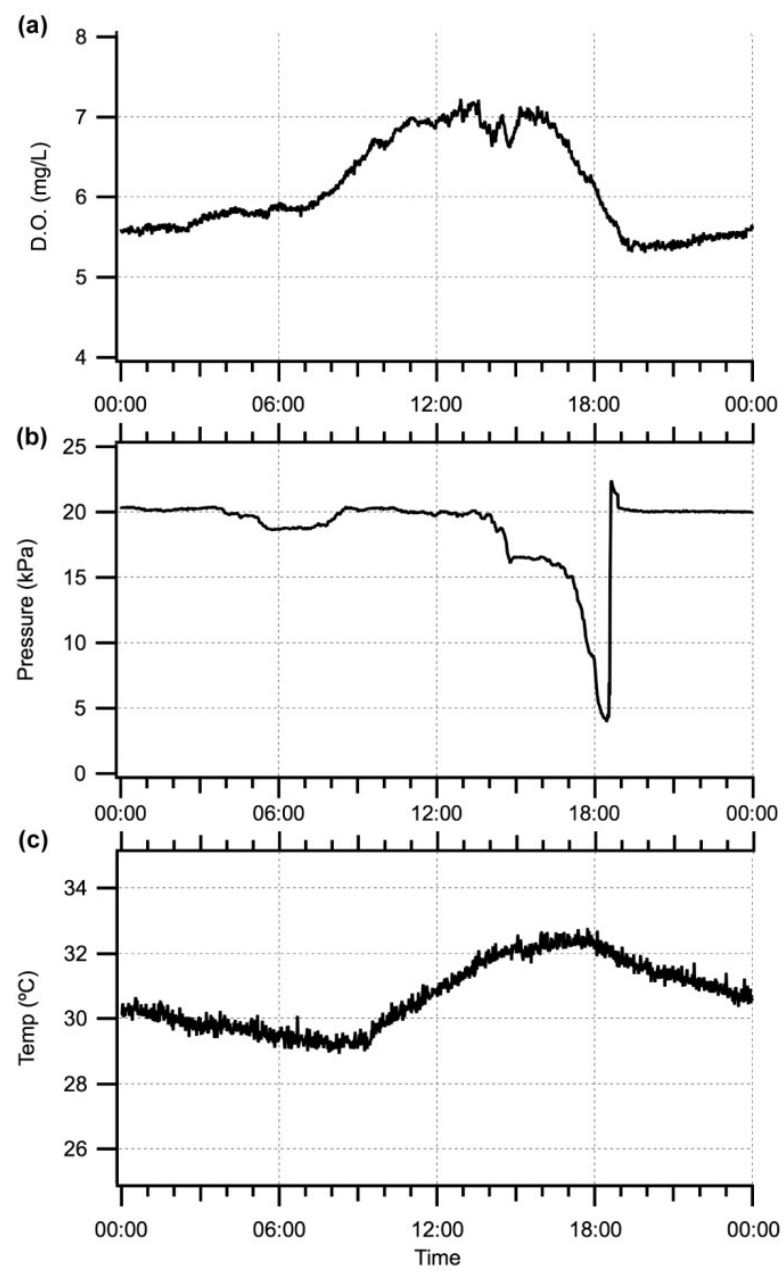

Figure 7. 24-hour measurements of (a) dissolved oxygen, (b) air pressure in the blower pipe, and (c) water temperature in a fish tank. Both dissolved oxygen and temperature increase during the day and decrease overnight due to oxygen production

by the photosynthesis of algae and to the absorbed daylight. Effects of maintenance work in the air-blower pipe can be observed as pressure fluctuates. 
Other sensors implemented in the system include a water pressure sensor at the base of the biological filter which is proportional to the water level. This level can signal anomalies in the recirculating pump behavior, reflected as changes in the water level of the biological filter or a problem in the overall adjustment of valves in the system. The current in the recirculating pump is also monitored. It can be used to monitor power consumption in the pumps and detect power anomalies that can be related to the power source or with the pump itself. Those signals could indicate anomalous power consumption of the recirculating pump that might precede a failure.

The monitoring system can be expanded to include more sensors and can be used to connect actuators. The two-digital input/output lines available in the module external connector can be used to control devices such as blowers or pumps to integrate an aquaculture monitor and control system.

\section{Conclusions}

This work presents the design and implementation of ZigBee enabled wireless sensor network modules. It remarks the benefits that a monitoring system can produce to a recirculating aquaculture unit and demonstrates an application of the ZigBee technology to aquaculture by designing, building and implementing a wireless sensor network for monitoring an aquaculture research facility.

The system monitors some of the relevant variables in recirculating aquaculture in real time and sends alerts when a reference limit has been reached. By alarming the system administrator, immediate actions can be taken to correct the situation and to reduce the risk of increased mortality or even production loss in the high fish densities typically handled in recirculating systems. Increases in productivity might also be expected since the data are taken in real time and actions can be taken immediately to correct deficiencies. In addition, the production history could increase the brand value certifying the production conditions of the fish at all times. The monitoring system can be expanded to become a monitor and control system to implement energy savings in the future.

\section{Acknowledgements}

The authors want to thank M. Vela for his help and technical support assembling and deploying the monitoring system and A. Repetto for his advice on the development of the Web interface.

\section{References}

[1] N. Shnel et al., "Design and Performance of a Zerodischarge Tilapia Recirculating System", Aquacultural Eng. vol. 26, no. 3, pp. 191-203, 2002.

[2] M. B. Timmons and J. E. Ebeling, "Recirculating Aquaculture". New York, Cayuga Aqua Ventures, 2007.

[3] S. Han et al., "Design of Environment Monitoring System for Aquaculture Farms", Proceedings of the 2007 Frontiers in the Convergence of Bioscience and Information Technologies. Jeju Island, Korea, IEEE Comp. Soc., 2007, pp. 889-893.

[4] V. C. Gungor and G. P. Hancke, "Industrial Wireless Sensor Networks: Challenges,Design Principles, and Technical Approaches". IEEE Trans. Ind. Elec., vol. 56, no.10, pp. 1265- 1282, 2009.

[5] F. Ding et al., "A GPS-enabled Wireless Sensor Network for Monitoring Radioactive Materials", Sensors and Actuators A, vol.155, no. 1, pp. 210215, 2009.

[6] R. Singh et al., "Pressure Monitoring in Wireless Sensor Network Using Zigbee Transceiver Module", Proceedings of the 2nd ICCCT-2011 International Conference on Computer \& Communication Technology. IEEE Conf. Pub., pp. 225-229, 2011.

[7] X. Zhu et al., "A Remote Wireless System for Water Quality Online Monitoring in Intensive Fish Culture", Computers and Electronics in Agriculture, vol. 71, sup. 1, pp. S3-S9, 2010.

[8] M. López et al., "Wireless Monitoring of the pH, NH4+ and Temperature in a Fish Farm", Proceedings of the Eurosensors XXIII conference, Procedia Chem., vol. 1, no. 1, pp. 445-448, 2009.

[9] M. López et al., "IEEE 802.15.4 Based Wireless Monitoring of $\mathrm{pH}$ and Temperature in a Fish Farm", Sensor Letters, vol. 7, no. 5, pp. 861-868, 2009. 
[10] M. F. Zhang and L. Z. Wang, "A WSN-based Monitor System for Water Quality Combined With Expert Knowledge", Proceeding of the 2011 International Conference on Electronics, Communications and Control, Ningbo, China, IEEE Conf. Pub., pp. 105-108, 2011.

[11] M.L. Shan et al., "The Design of Wireless Sensor Node Based on Zigbee for Salinity Measurement". Proceeding of the 2011 International Conference on Electronics, Communications and Control, Ningbo, China, IEEE Conf. Pub., pp. 2355-2358, 2011.

[12] P. Baronti et al., "Wireless Sensor Networks: A Survey on the State of the Art and the 802.15.4 and ZigBee Standards", Comp. Comm., vol. 30, no. 7, pp. 1655-1695, 2007.

[13] I.F. Akyildiz et al., "Wireless Mesh Networks: a Survey". Comp. Net. vol. 47, no. 15, pp. 445-487, 2005.

[14] Z. Shelby and C. Bormann, "6LoWPAN: The Wireless Embedded Internet", Hoboken, N.J., Wiley, 2010, pp.1-24.

[15] S. Farahani, "ZigBee Wireless Networks and Transceivers", Newness-Elsevier, Burlington, MA, 2008. pp 329.

[16] X. Zhang, "Remote Monitoring System of Textile Equipments Based on ZigBee Technology". Adv. Mat. Res. vol. 472-475, pp. 174-177, 2012.

[17] X. Liu et al. "Design of the Clutch Friction Plate Temperature Acquisition System Based on Zigbee", App. Mech. and Mater. vol. 155-156, pp. 1102-1106, 2012.

[18] S. Chen et al. "Analysis of the Power Consumption for Wireless Sensor Network Node Based on Zigbee". Proc. Eng.. vol. 29, pp. 1994-1998, 2012.

[19] M. Terada, "Application of ZigBee Sensor Network to Data Acquisition and Monitoring", Meas. Sci. Rev., vol. 9 no.6, pp. 183-186, 2009.

[20] Atmel Corp., "ZigBit ${ }^{\mathrm{TM}} 2.4 \mathrm{GHz}$ Wireless Modules Datasheet", available from http://www.atmel.com, and "The ZigBee Specification", available from the ZigBee Alliance, http://www.zigbee.org/Specifications.aspx .

[21] Atmel Corp. “Atmel BitCloud Users Guide”, available from http://www.atmel.com .

[22] P. Horowitz and W. Hill, "The Art of Electronics". $2^{\text {nd }}$ Ed., Cambridge, UK, Cambridge University Press, 1989, pp. 351-466. 